EUROPEAN ORGANIZATION FOR NUCLEAR RESEARCH

CERN - SL DIVISION

CERN-SL-99-002 DI

\title{
Performance Related Measurements on LEP
}

\author{
S. Myers
}

\begin{abstract}
Measurements of the LEP beam parameters have been fundamental to performance improvements and this report describes the principles and techniques involved in the beam instrumentation systems of LEP.
\end{abstract}

Geneva, Switzerland

January 13, 1999 


\section{Contents}

1 Introduction $\quad 1$

2 Measurement techniques 2

3 Current transformer $\quad 2$

4 Measurements with the pick-ups $\quad 2$

4.1 Locating obstacles using the first-turn trajectory measurement . . . . . . . . . . 3

4.2 K Modulation . . . . . . . . . . . . . . . . . . . . . . 4

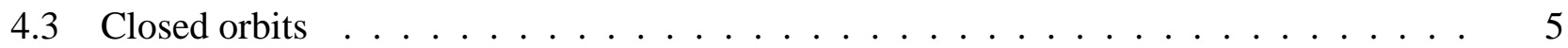

4.4 Momentum dispersion $\ldots \ldots \ldots \ldots \ldots \ldots$

5 The "1000-turns" facility $\quad 7$

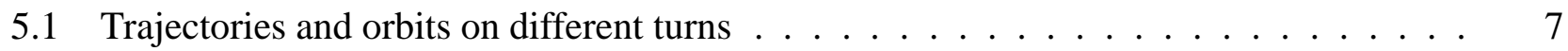

5.2 Betatron phase advance . . . . . . . . . . . . . . . . . 7

5.3 Evaluation of $\beta$ and $\alpha \ldots \ldots \ldots \ldots \ldots \ldots$

5.4 Phase-plane measurements . . . . . . . . . . . . . . . . . 9

5.5 Measurement of detuning . . . . . . . . . . . . . . . . . . 9

6 Frequency of oscillation $\quad 9$

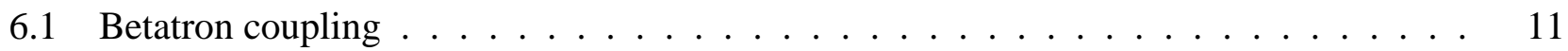

6.2 Chromaticity and central frequency . . . . . . . . . . . . . . . . 12

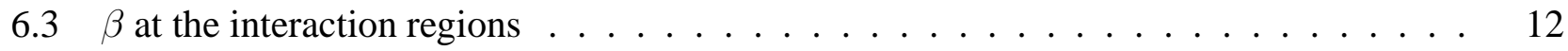

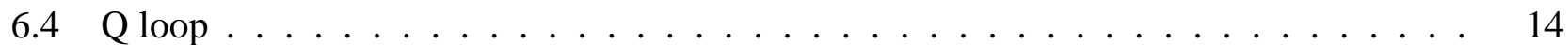

7 Emittance measurement $\quad 14$

7.1 Tune scans . . . . . . . . . . . . . . . . . . . . . . 15

8 Measurements in collision $\quad 16$

8.1 Beam-beam deflection $\ldots \ldots \ldots \ldots \ldots$

9 Acknowledgments $\quad 16$

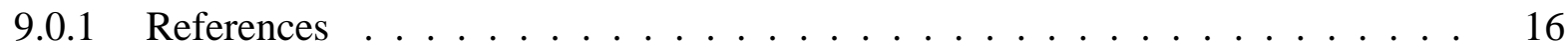




\section{Introduction}

The CERN Large Electron Positron (LEP) collider is a $26.6 \mathrm{~km}$ circumference $\mathrm{e}^{+} \mathrm{e}^{-}$storage ring which has, until the end of 1995, operated with 4 and 8 bunches per beam in an energy range of 20 to $50 \mathrm{GeV}$ (see conference reports, [1], [2], [3]' [4]).

The LEP energy upgrade was foreseen to allow study of $\mathrm{W}$ pairs at energies above $90 \mathrm{GeV}$ per beam, and involves a total of 288 new superconducting (sc) cavities with design gradients of $6 \mathrm{MV} / \mathrm{m}$.

In any collider the most important performance parameter is the luminosity (L) which defines the number of events seen per second at each interaction region. i.e.

$$
\frac{d N_{p}}{d t}=\sigma_{p} L
$$

where $\sigma_{p}$ is the cross-section of the process.

For $\mathrm{e}^{+} \mathrm{e}^{-}$colliders the general equation for luminosity is

$$
L=\frac{i_{e} i_{p} k_{b} \exp \left\{-\frac{\left(\bar{x}_{e}-\bar{x}_{p}\right)^{2}}{2\left(\sigma_{x e}^{2}+\sigma_{x p}^{2}\right)}-\frac{\left(\bar{y}_{e}-\bar{y}_{p}\right)^{2}}{2\left(\sigma_{y e}^{2}+\sigma_{y p}^{2}\right)}\right\}}{2 \pi f_{r e v} e^{2} \sqrt{\left(\sigma_{x e}^{2}+\sigma_{x p}^{2}\right)\left(\sigma_{y e}^{2}+\sigma_{y p}^{2}\right)}}
$$

where $i_{e}$ and $i_{p}$ are the electron and positron bunch currents,

$k_{b}$ is the number of bunches per beam,

$\left(\bar{x}_{e}-\bar{x}_{p}\right),\left(\bar{y}_{e}-\bar{y}_{p}\right)$ are the horizontal and vertical distances between the centres of gravity of the electron and positron beams,

$f_{\text {rev }}$ is the revolution frequency,

and $\sigma_{x e}, \sigma_{x p}, \sigma_{y e}$, and $\sigma_{y p}$ are the rms horizontal and vertical beam dimensions of the electron and positron beams, and given by

$$
\sigma_{\text {tot }}^{2}=\sqrt{\sigma_{\beta}^{2}+\sigma_{\epsilon}^{2}}=\sqrt{\beta \varepsilon+\left(D \frac{\sigma_{\epsilon}}{E}\right)^{2}}
$$

where $\varepsilon$ is the betatron emittance, $\mathrm{D}$ is the momentum dispersion, and $\sigma_{\epsilon} / E$ is the energy spread (more accutately the momentum spread).

In order to maximize the luminosity it is clear from equation (2) that the following parameters must be optimized:

- Maximize the electron and positron bunch currents. This involves optimization of the injection efficiency, avoidance of all types of resonances, and taking the necessary precautions for increasing the thresholds of transverse and longitudinal instabilities.

- Maximize the number of bunches per beam $\left(k_{b}\right)$. This may incur limitations to the total beam current, unwanted beam-beam collisions and the beam-beam tune shift and spread.

- Collide the beams head-on in all interaction regions: i.e. make all separations zero. This necessitates measurement of the separations, orbit differences between electrons and positrons and the possibility of correcting differentially electron and positron orbits.

- Minimize the rms beam dimensions. This requires avoidance and minimization of resonances, correction of betatron coupling, minimization of residual momentum dispersion, and correction of optical functions.

The instruments used and the measurement techniques employed for this performance optimization are discussed in the following sections. 


\section{Measurement techniques}

The measurement of the properties of a charged particle beam may be made by two main techniques.

Firstly the direct electro-magnetic field associated with the beam may be measured and processed: examples of this are the beam current, the longitudinal profile of the bunch, and the centre of gravity of the charge of the bunch (orbit).

Secondly, primary or secondary particles associated with the beam may be detected: examples of this are the detection of the photons associated with the synchrotron radiation, primary particle losses (loss monitors), scattered particles (background), and back-scattered photons (polarization). In addition the luminosity may be measured by measuring the rate of certain events such as Bha-Bha scattering from the interaction point.

\section{Current transformer}

One of the most simple to understand and useful instruments in an accelerator is the current transformer which uses toroids to couple to the beam current. The measured current is given by

$$
i_{b}=\frac{d Q}{d t}=n_{b} e f_{r e v}
$$

In addition the same measurement differentiated wrt to time gives the beam lifetime

$$
\frac{d i_{b}}{i_{b} d t}=\frac{1}{\tau_{b}}
$$

In LEP the total circulating beam current is measured [5] with one current transformer and the current in each bunch is measured with a second current transformer. The measured current per bunch is used to control injection so as to maintain approximately equal the current in all bunches during accumulation. This instrument, the "Bunch Current Equaliser" is essential when accumulating to the highest possible currents.

The measurement of the beam lifetime [6] is also crucial for tuning the machine parameters for optimising the performance.

\section{Measurements with the pick-ups}

When a charged beam passes between two parallel conductors (or pick-ups), it induces a difference voltage $\left(\mathrm{v}_{b}\right)$ on the plates given by

$$
\mathrm{v}_{b} \propto n_{b} \Delta u
$$

where $\Delta u$ is the displacement of the centre of the beam wrt the centre of the plates. In addition, to first approximation, the sum signal is proportional to the beam intensity.

In LEP there are 504 "button-type" pick-ups located around the circumference. Each pick-up consists of four electrodes (buttons) which are displaced with respect to the median plane in order to avoid perturbation of the electro-magnetic signals caused by synchrotron radiation. Combination of the signals from the four electrodes using a suitable algorithm allows evaluation of the position of the centre of charge of the beam with respect to the electrical centre of the pick-up, in both the horizontal and the vertical plane. Those pick-ups which are very close to the interaction point have special electronics attached to each of the four electrodes. This allows measurement of the sum signal from all four electrodes giving an evaluation of the beam intensity as well as the beam centre of charge at these particular pick-ups. 
The electro-magnetic signal associated with each beam of particles is processed at each pick-up on each turn and stored in a memory buffer large enough to contain data for more than 1000 turns. This software [7] allows measurement of

- intensity variations, using the sum of the signals on all pick-ups

- injection trajectories

- closed orbits: the precision of this measurement is significantly improved by averaging the signal at each pick-up over a large number of revolutions (typically 225)

- measurement of the optics parameters by exciting a coherent oscillation in the beam (see later).

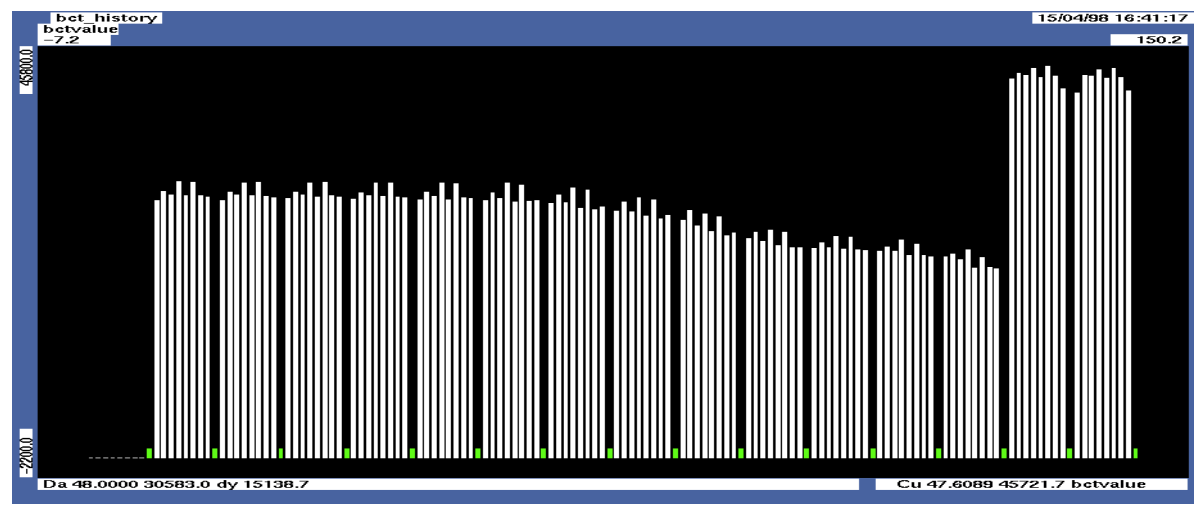

Figure 1: Sum signal

Figure 1 shows the sum signal (as a function of time) of the pick-ups which are near the interaction region. The eight vertical bars correspond to the measured sum signal (intensity) on each octant of the circumference.. It may be seen from this Figure that the intensity starts to decrease after around seven to eight turns (a turn is a group of eight vertical lines followed by a space) and a second injection is made after 13 turns. This type of measurement is also very useful for narrowing down the region in a search of possible obstacles in the beam path: a loss can be seen between two successive octants.

\subsection{Locating obstacles using the first-turn trajectory measurement}

One of the most critical phases for an accelerator is to establish a first circulating beam after a long shutdown. When the beam is made to circulate for a reasonable number of turns it is a clear sign that the aperture is free of obstacles and that the optics configuration has no serious errors such as magnets with inverted polarity. For the rare occasions that the beam does not make more than a turn or two it is crucial to have a reliable diagnostic tool which can be used to locate the azimuthal location of the fault. In LEP the pick-up system has been used on several occasions to locate the position of faults such as a collimator protruding into the aperture, closed vacuum valves, and even beer bottles blocking the vacuum pipe. The technique is simple. A measurement of the first turn trajectory is made, then a known kick is added just after the injection region and a second trajectory is measured. The difference between the two trajectories is simply the influence of the known kick and when displayed, normalized by the $\beta$ function, should show a sampled sinusoidal oscillation. Any divergence from the sinusoidal oscillation indicates the location of the fault.

Figure 2 shows the results of such a measurement. Until a certain azimuthal location the trajectory follows a sampled sinus and from the location marked "QL10.L1" the oscillation is perturbed. On this occasion the vacuum chamber was opened to air and the offending obstacle was found and removed. 


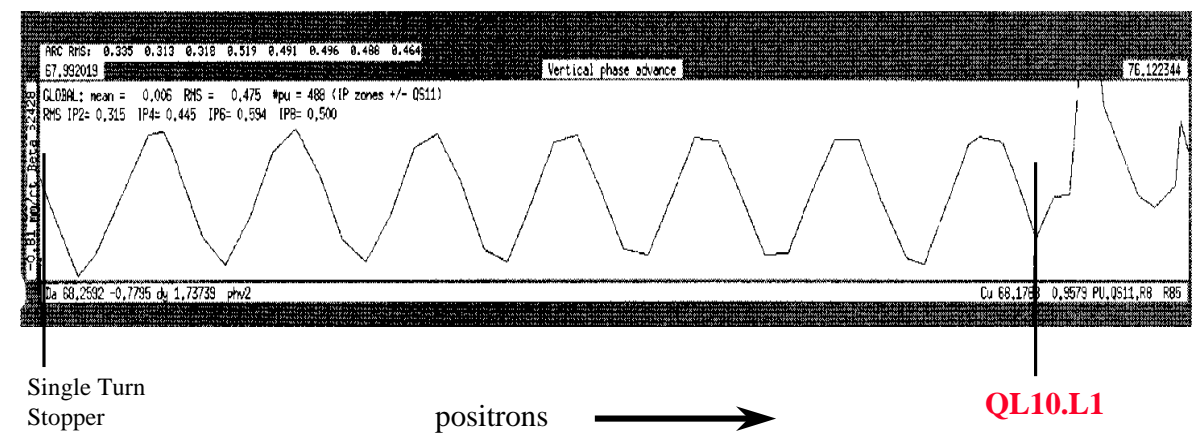

Figure 2: Locating obstacles in the beam pipe

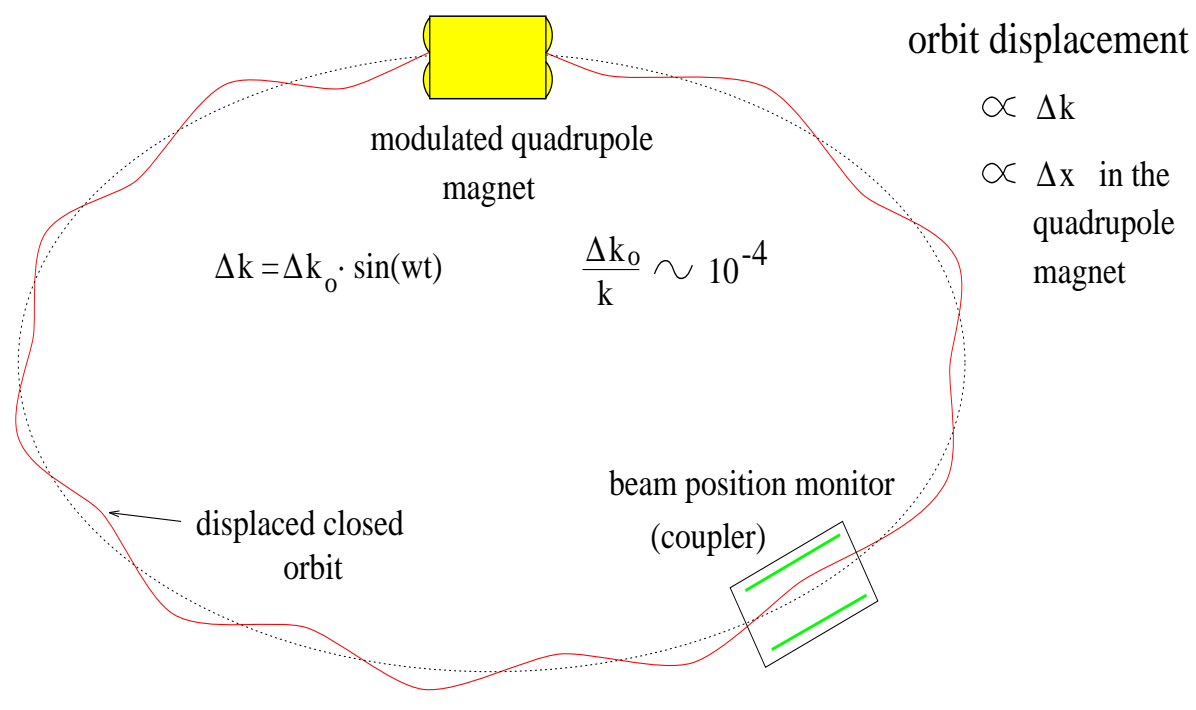

Figure 3: Schematic of principle of K-Modulation

\subsection{K Modulation}

The main objective of measurement and correction of the closed orbit is to minimize the offset of the beam with respect to the magnetic centres of the quadrupoles. In practice, since the orbit can only be measured at the pick-ups, the closed orbit correction procedure minimizes the rms offset of the beam with respect to the electrical centre of the pick-ups. Consequently in order to meet the primary objective every effort is made to ensure that the electrical centre of the pick-up is identical to the magnetic centre of the quadrupoles. In LEP for example, the pick-ups are physically attached and aligned to the nearby quadrupole. Nevertheless errors do occur in construction and installation and it is very important to be able to measure and correct [8] any deviations between the pick-ups and quadrupoles.

A schematic which explains the technique [9] used to measure such deviations is shown in Figure 3. The gradient of each quadrupole is modulated (in the case of LEP by using a "back-leg" winding) at a low frequency of around 10 to $20 \mathrm{~Hz}$. The resulting modulation (at this frequency) of the closed orbit is measured using a high sensitivity monitor. Clearly the beam is centered in the quadrupole when the Fourier component of the measured orbit modulation is minimized. The average orbit as measured at the pick-up attached to the quadrupole is also recorded. The beam can then be displaced at the location of the quadrupole and attached pick-up until the measured Fourier component of the orbit modulation is minimum.

Figure 4 shows an example of such a measurement. The Fourier component of the modulation is plotted as a function of the measured average orbit at the adjoining pick-up. This plot, which was performed for several different modulation frequencies shows that the minimum Fourier amplitude occurs when the pick-up indicates $-0.5 \mathrm{~mm}$, i.e. the offset is this value. 


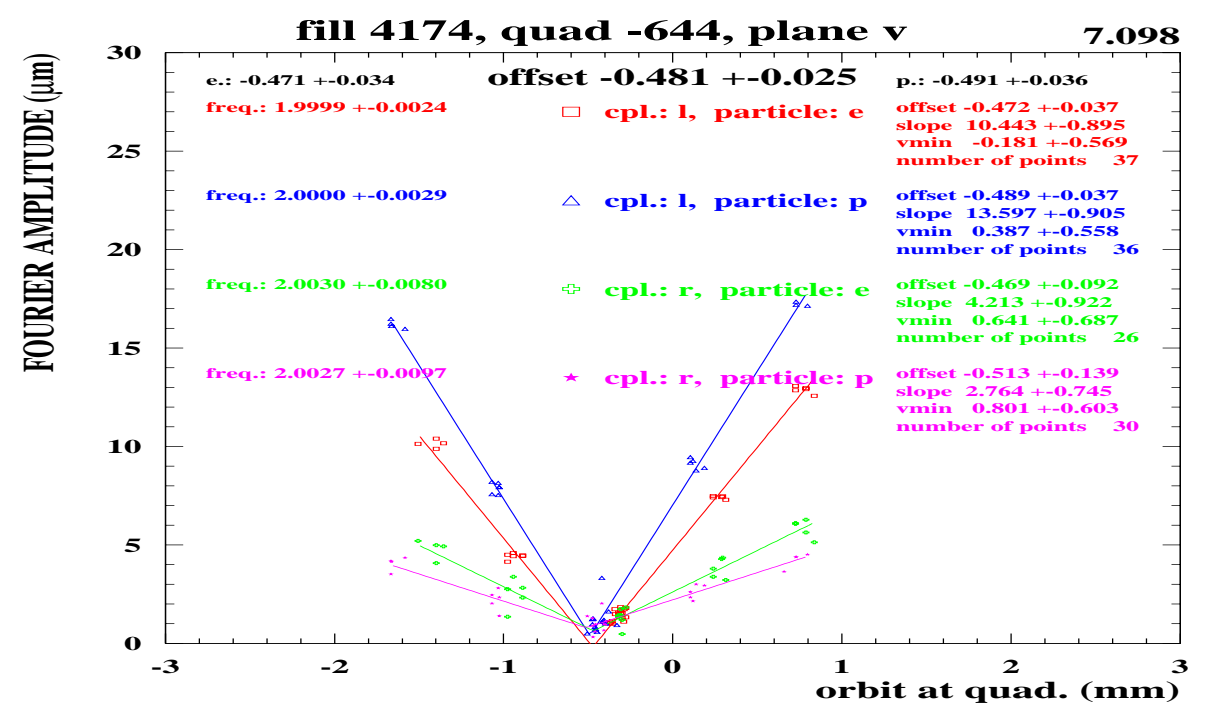

Figure 4: Measurement of offset between PU and Quad using K-Modulation
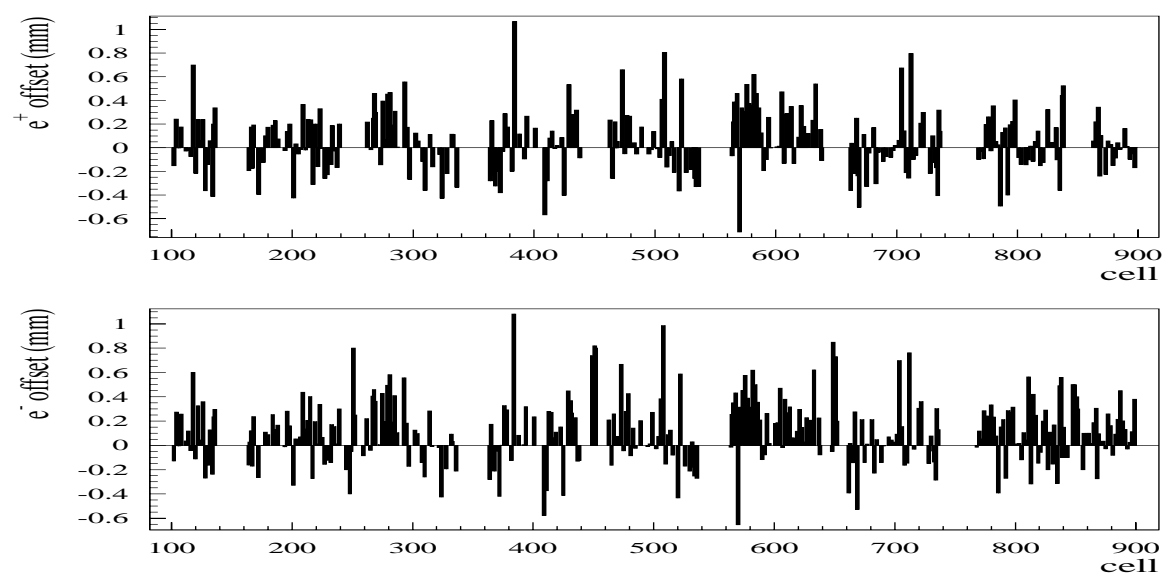

Figure 5: Offsets as measured at PUs around the LEP circumference

This procedure was performed on all LEP pick-ups and for the electron and positron beams. The measured offsets are shown in Figure 5 which shows maximum offsets of around $1 \mathrm{~mm}$. The measured values are then made available to the closed orbit measurement software which compensates for the offsets. It may be seen that there are slight differences in the measured offsets for electrons and positrons. This is not yet fully understood.

\subsection{Closed orbits}

Figure 6 shows a typical measured orbit which used averaging over many turns (the circumference is "linearized" for display purposes, starting at IP1 on the left going to IP8 on the right). This type of measurement (and subsequent orbit correction) is performed many times during a physics run and is crucial to the performance of the machine. In order to optimize the performance of LEP the rms deviations of the closed orbit must be corrected [10] to be well below $1 \mathrm{~mm}$ and when very high luminosities are registered after a particular orbit correction this orbit is declared "golden". In subsequent runs the closed orbits are corrected back to the most recent "golden" orbit.

\subsection{Momentum dispersion}

The data acquisition system for the closed orbit allows storage and manipulations of orbits measured at different times. This facility is used for the measurement of the momentum dispersion. The displace- 


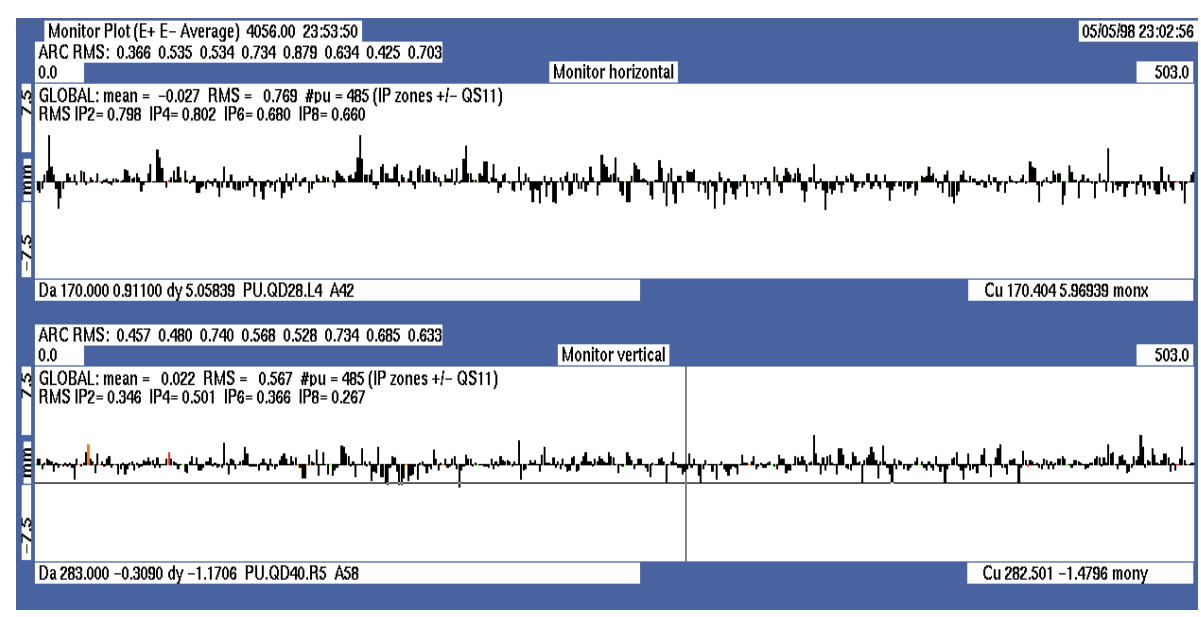

Figure 6: Measured closed orbit

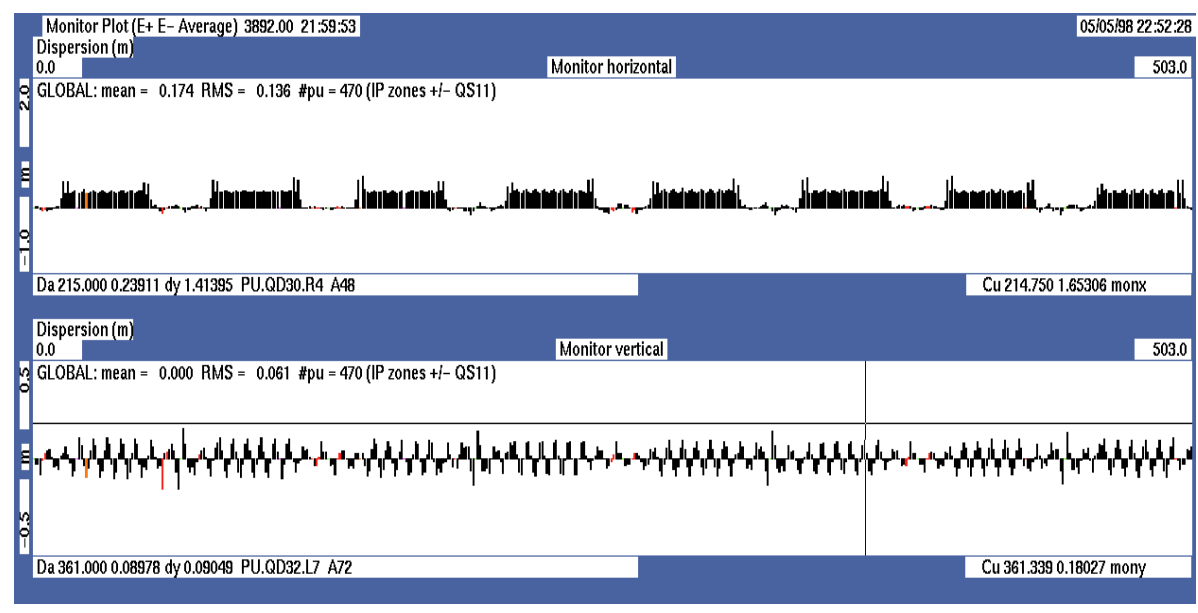

Figure 7: Measurement of momentum dispersion

ment at each pick up is given by

$$
u=u_{c o}+D_{u} \frac{\delta p}{p}=u_{c o}+\frac{D_{u}}{\eta} \frac{\delta f}{f}
$$

where $u_{c o}$ is the displacement due to the closed orbit,

$D_{u}$ is the momentum dispersion at the location of the pick-up,

$f$ is a harmonic of the revolution frequency e.g. the RF frequency, and $\eta$ is given by $\frac{1}{\gamma_{t}^{2}}-\frac{1}{\gamma^{2}}$.

Clearly by subtracting orbits made at different $\frac{\delta p}{p}$ (by changing the RF frequency), a measurement of the dispersion at each pick-up is possible, i.e.

$$
u_{i 1}-u_{i 2}=-\frac{D_{u i}}{\eta} \frac{\Delta f}{f} .
$$

Figure 7 shows a typical measurement of the dispersion using the subtraction technique. The upper plot is the measurement in the horizontal plane showing that the dispersion is high and constant through the arcs and low but with some residual horizontal dispersion in the eight straight sections which should be dispersion-free. The residual vertical measurement in the lower plot is shown on a different scale and indicates the vertical dispersion around the LEP circumference. The residual dispersions are minimized at the locations of the RF cavities to reduce the excitation of synchro-betatron resonances and at the interaction points to reduce the overall beam size and the influence of beam-beam synchro-betatron resonances. 


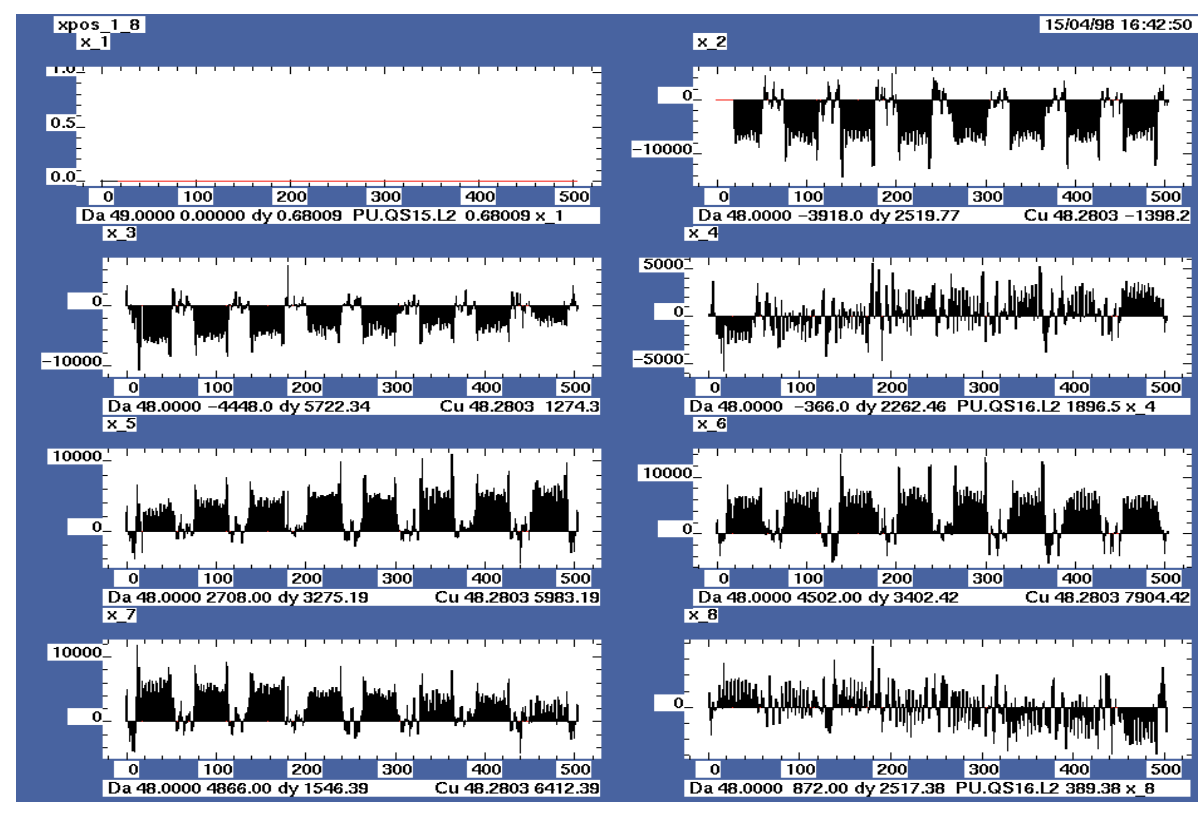

Figure 8: Orbits on different turns; Synchrotron injection

\section{The "1000-turns" facility}

\subsection{Trajectories and orbits on different turns}

The Beam Orbit Measurement (BOM) system can also be triggered to measure orbits or trajectories on pre-programmed timing signals. This facility used in conjunction with the possibility of storing 1024 measurements has many useful applications. Two frequently used and useful examples are measurements of trajectories at injection and measurement of orbits during the energy ramp. Figure 8 shows measured trajectories of the positron injected beam for the first eight turns after injection. In this particular case "synchrotron injection" was being studied whereby the beam is injected with an energy offset into the outside of the RF bucket and oscillates and damps in synchrotron phase space. One can easily see from the measured trajectories via the dispersion in the arcs that the beam is clearly making energy oscillations at an approximate rate of one synchrotron oscillation every eight turns i.e. Qs $\simeq 0.12$. The multi-turn measurement technique has been used extensively for optimizing synchrotron injection and for the measurement of orbits (and subsequent correction) during the energy ramp.

\subsection{Betatron phase advance}

Another invaluable use of the "1000-turns" facility is the measurement of optical parameters ( $\beta$ functions etc.) along the LEP circumference for comparison (and correction) to the theoretical values. This technique involves transverse excitation of the beam in order to maintain a coherent betatron oscillation whilst measuring the beam position at each pick-up during 1024 successive revolutions [11], [12]. The "1000-turns" data are then harmonically analyzed to give the amplitude and phase of the oscillation at each pick-up. The relative betatron phase advance between each pick-up can then be plotted and compared with the theoretical values. Figure 9 (upper trace) shows an example measurement in the vertical plane for an optics in LEP where the theoretical phase advance per cell in the arcs is $60^{\circ}$. The vertical range in this plot is modulo $360^{\circ}$. Consequently if the phase advance between pick-ups in the arcs was exactly $60^{\circ}$ the measurement would give six equally spaced groups of dots giving the impression of a straight horizontal line. The actual measurement indicates this behavior approximately in the eight arcs. The deviations from the theoretical phase advances are indicated in the lower plot of Figure 9. It may be seen on the right hand side of this plot, at pick-up numbers 450 to 475, that the "errors" have a step function. This is simply due to the fact that the turn number for this group of pick-ups is wrong 


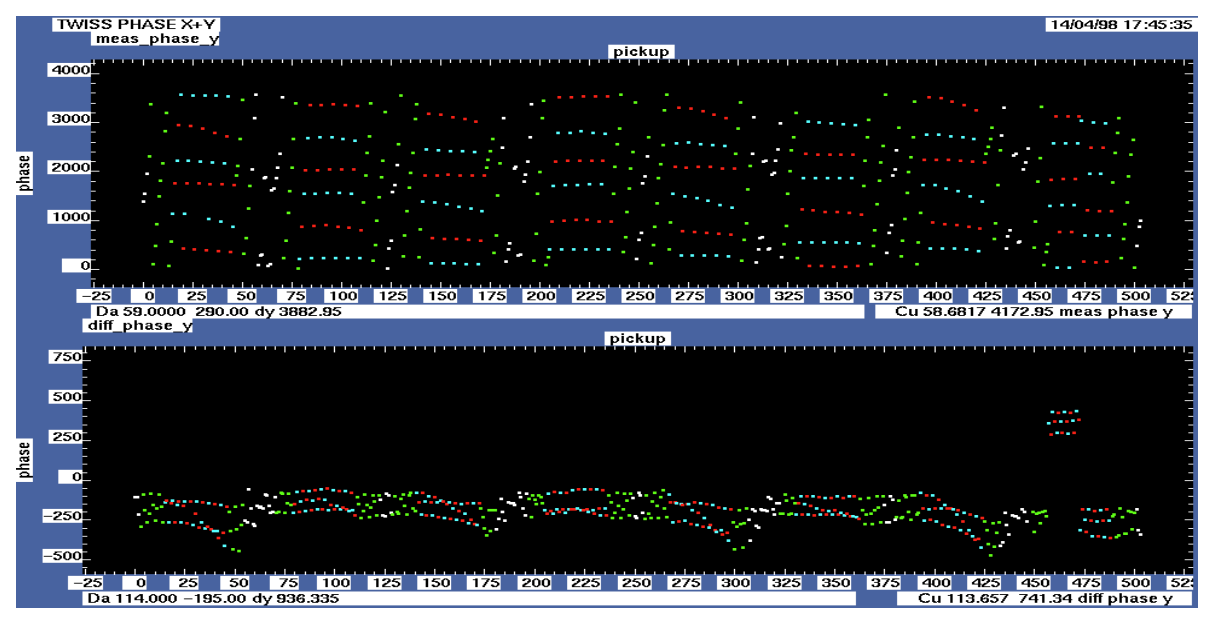

Figure 9: Measurement of the phase advance in the vertical plane (10 units $=1$ degree $)$.

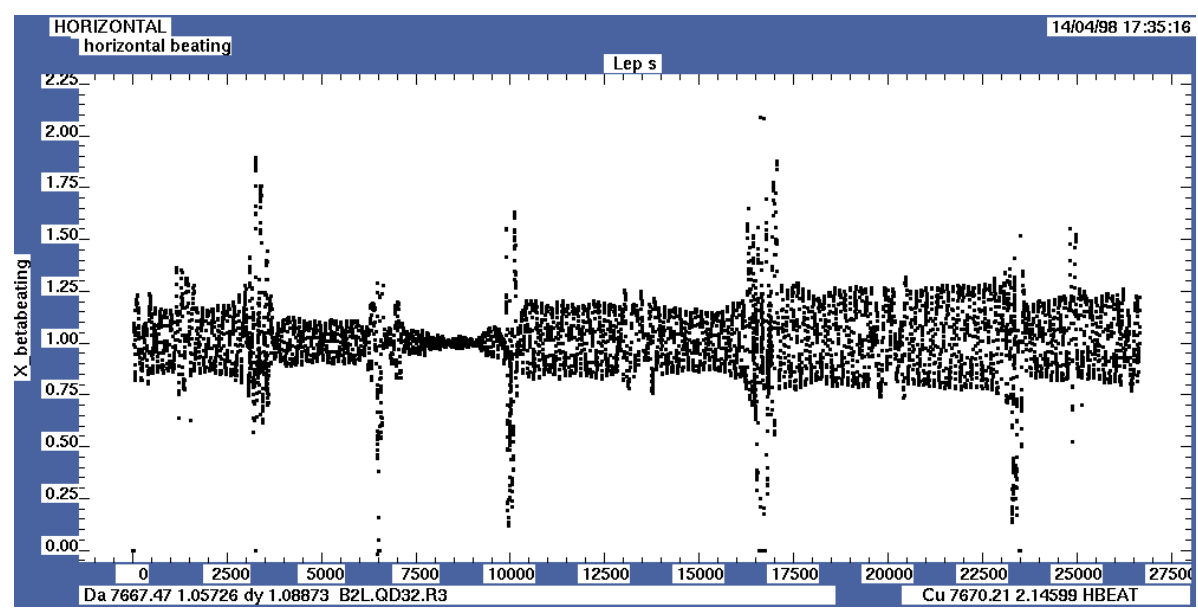

Figure 10: Measured beta beating in the horizontal plane. LEP is shown from IP1 to IP8: the low-beta insertions are in the even IPs.

by a single turn thereby changing the phase advance by the non-integer part of the vertical tune. This example highlights the importance of measuring all pick-up signals on the same turn.

\subsection{Evaluation of $\beta$ and $\alpha$}

The measured phase advances between the pick-ups can be used to evaluate the optics parameters at any azimuthal position around the circumference [13]. Taking a given pick-up and the ones adjacent and using the three measured phase advances along with the theoretical phase advances coming from a tracking programme such as MAD allows evaluation of the ratio of the real $\beta$ value to the theoretical one. This procedure is correct provided the perturbation which is causing the $\beta$ beating is outside the three chosen pick-ups. The values at the pick-ups are obtained by "advancing" around the circumference from one central pick-up to the next, and values between the pick-ups are obtained by using the standard transfer matrices. In general the perturbations arise in the insertion regions so this procedure predicts the correct values of $\beta$ in the machine arcs. The beta functions at the insertion quadrupoles are measured by a separate procedure (see later).

Figure 10 shows an example of a measurement of $\beta$ beating in the horizontal plane. The largest beating, as expected, comes from the four low- $\beta$ insertions with some beating in the high- $\beta$ insertions.

Figure 11 shows a zoom of the $\beta$ measurement in an arc. The upper plot shows the regular $\beta$ variations in the arc cells and the lower plot shows the relative $\beta$ beating with respect to the theoretical values. In this case it can be seen that the measured beating is of the order of $\pm 10 \%$. 


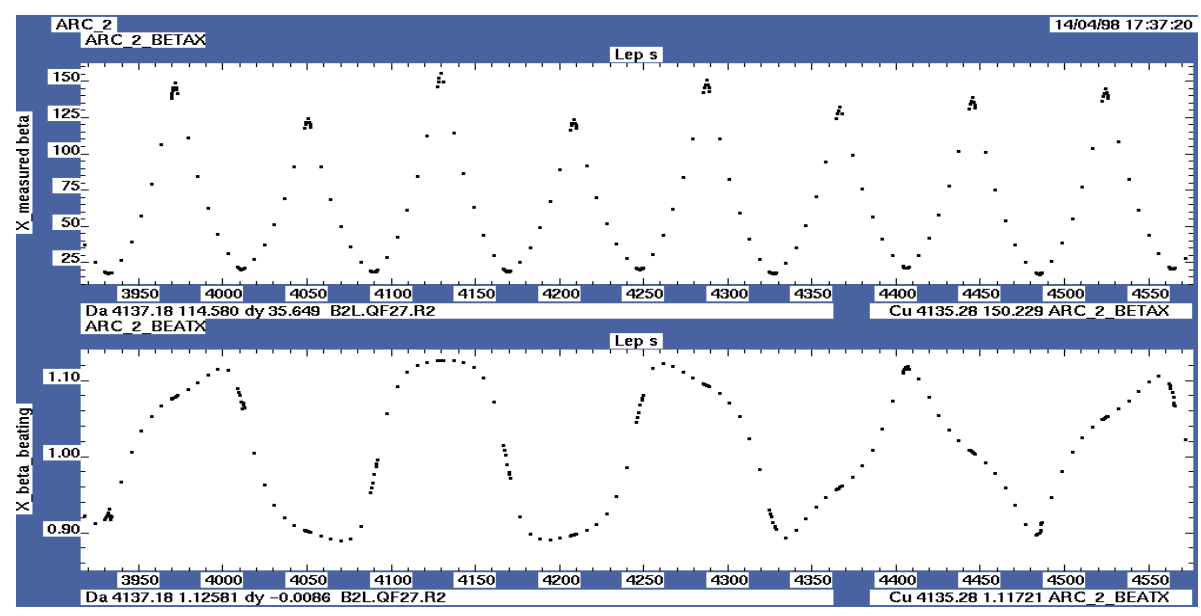

Figure 11: Measurement of horizontal beta beating in the arcs

\subsection{Phase-plane measurements}

Inspection of the motion of the centre of charge of beams in phase space ( $u$ vs $u^{\prime}$ ) is often helpful in understanding the related beam dynamics. For example, resonances can be more easily identified. The more normal way of observing phase plane motion is to excite a coherent oscillation and measure the position of the beam at two pick-ups which are approximately $90^{\circ}$ apart in betatron phase space. In LEP the 1000-turns facility is used for this purpose in the following way. At a given pick-up, the beam positions measured over 1000 turns are stored and the measurement on each successive turn is plotted against the position on the previous turn [15]. In the case where the non-integer part of the tune is 0.25 this gives a perfect phase-plane plot. However variations from tunes of 0.25 simply distort the plot without obscuring many details. Figures 12 and 13 show examples of this type of measurement when the beam is close to a third- and seventh-order resonance respectively.

These types of measurements are frequently used when measuring the dynamic aperture by exciting large coherent oscillations. The detuning with amplitude often drives the beam onto a lower-order resonance.

\subsection{Measurement of detuning}

Another useful application of the 1000-turns technique is the measurement of the variation of the betatron tune as a function of betatron amplitude [16]. For this measurement the beam is "kicked" to have a large betatron amplitude of several millimeters and then the amplitude is allowed to decrease under the influence of synchrotron or chromatic damping. The decaying beam displacement is recorded using the 1000 turns facility. An example is shown in Figure 14.

Samples of data taken over say 50 turns are then harmonically analyzed to give the tune and the invariant betatron amplitude. An example of such a measurement is shown in Figure 15. This technique has been successfully used to confirm the theoretical predictions of the "anharmonicity" $(\delta Q / \delta \epsilon)$ which is an important factor in the available dynamic aperture.

The same procedure is also used to measure the damping time of coherent oscillations. A measurement made at $60 \mathrm{GeV}$ per beam is shown in Figure 16.

\section{Frequency of oscillation}

Many useful and interesting beam parameters can be attained by measuring the response of the beam to excitation. For example, if the beam is excited transversely with band-limited noise (or a swept frequency) the response in frequency domain from a pick-up allows determination of the transverse tunes. In general when the non-integer betatron tune is $q$, then the beam-excited response shows a maximum 


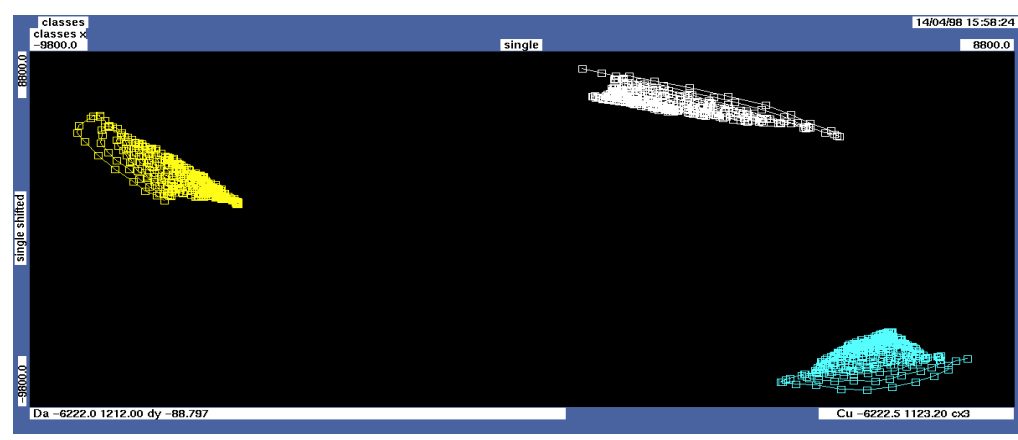

Figure 12: Third-order resonance

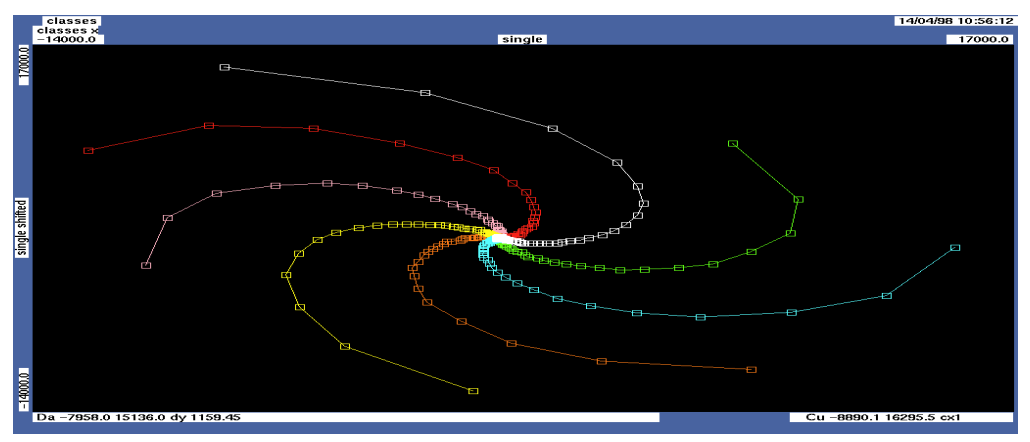

Figure 13: Phase Plane of 7th-order resonance

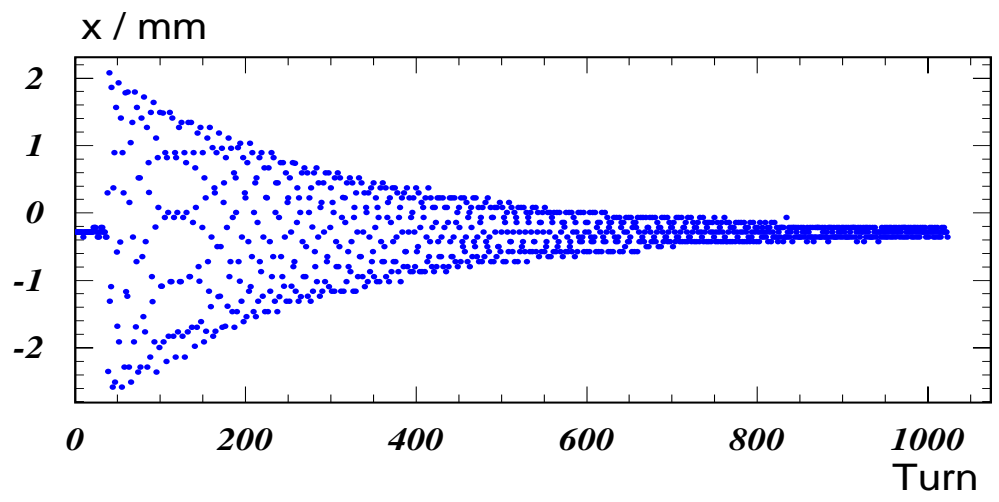

Figure 14: Horizontal amplitude as function of number of turns

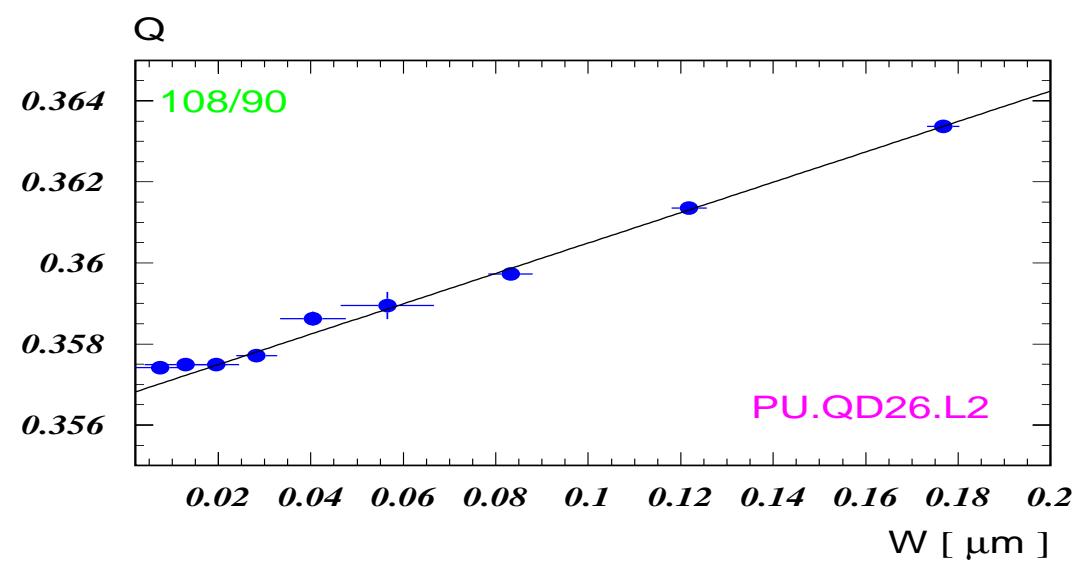

Figure 15: Dependence of vertical tune on normalised amplitude 


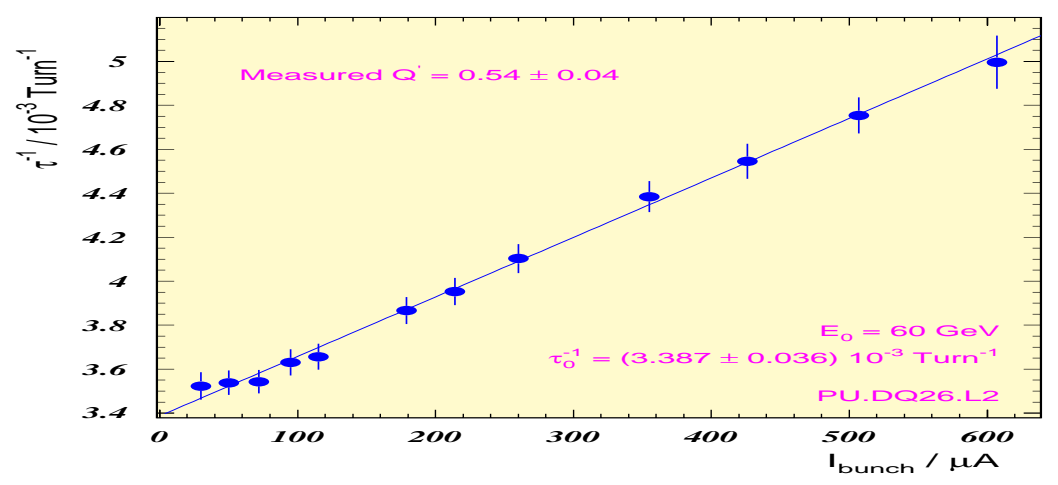

Figure 16: Damping coefficient as function of bunch current

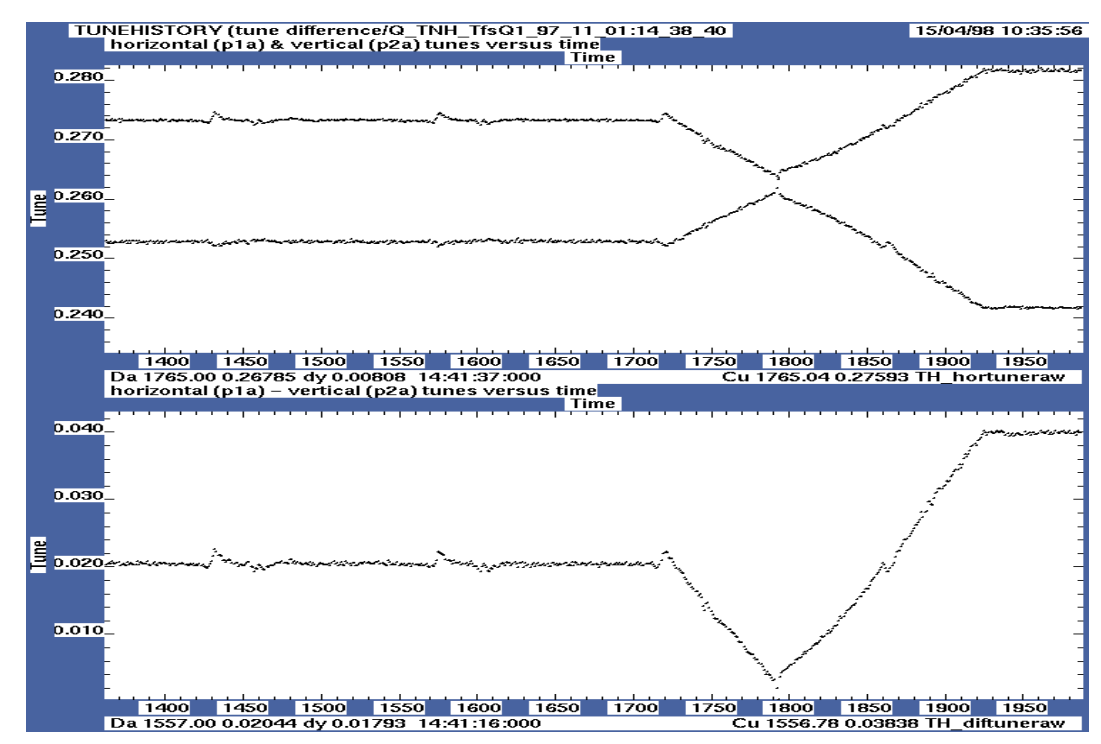

Figure 17: Closest-tune approach showing significant betatron coupling

in amplitude at a frequency of $q f_{r e v}$ and the phase response traverses $0^{0}$ at the same frequency. Consequently if there is a feedback loop which maintains the phase response at $0^{0}$ by controlling the excitation frequency, then the $q$ can be continuously measured from the value of the excitation frequency. This is called a beam phase-locked loop and has many applications in beam diagnostics and measurements.

\subsection{Betatron coupling}

A quantitative measure of the amount of emittance coupling between the horizontal and vertical planes is given by the minimum tune difference which can exist between the two planes before the tunes lock onto a single value. This quantity can be measured by continuously monitoring the tunes in the two planes as the tunes are driven through each other. An example of such a measurement is shown in Figure 17 where the upper plot shows the horizontal (initially upper trace) and vertical tunes monitored as a function of time. Towards the end of the plot the horizontal and vertical tunes are ramped downwards and upwards respectively. When the tunes are approximately equal, they lock on to each other. The lower trace is the difference between the tunes and shows a minimum value which is not zero. This value is a measure of the betatron coupling .

Figure 18 shows a similar plot after the correction of the coupling using skew quadrupoles. The minimum difference in tunes is clearly reduced. 


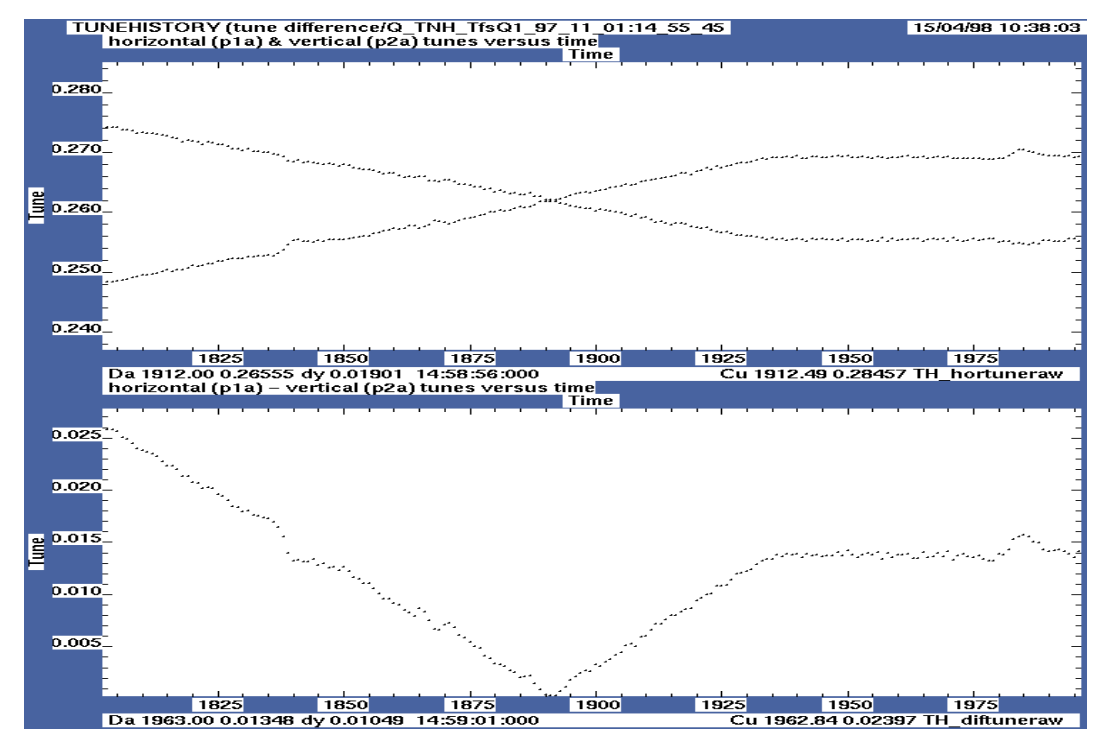

Figure 18: Measurement of coupling after correction.

\subsection{Chromaticity and central frequency}

The chromaticity may be measured by varying the RF frequency and measuring the resulting variation in the tune. i.e.

$$
\frac{\Delta Q}{\Delta p / p}=Q^{\prime}=-\eta \frac{f_{r f}}{\Delta f_{r f}} \Delta Q
$$

Figure 19 shows the measured modulation in the horizontal (upper trace) and vertical tunes as a result of a sinusoidal modulation of the RF frequency [17]. The peak-to-peak values of the tune modulations are proportional to the chromaticity.

The chromaticity may of course be measured by varying the frequency linearly and measuring the resulting variation in the tune. A range of such measurements with different values for the chromaticity is shown in Figure 20. The frequency at which all the linear fits pass through the same point clearly corresponds to the magnetic centre of the sextupoles [18]. In LEP the knowledge of this frequency is crucial for calibration of the beam energy. It is also important for the evaluation of the frequency shift required to control the emittance (see later).

\section{3 $\beta$ at the interaction regions}

Changing the normalized gradient $(k)$ in a quadrupole produces a tune shift. i.e.

$$
\Delta Q \backsim-\frac{\beta}{4 \pi} k l
$$

where $l$ is the length of the quadrupole and $\beta$ is the betatron value at it.

Between the final quadrupoles in a low $\beta$ insertion, where there are no quadrupoles, the $\beta$ function varies quadratically with the distance $(s)$ from the location of the minimum $\beta$. i.e.

$$
\beta(s)=\beta^{*}\left(1+\frac{s^{2}}{\beta^{*}}\right)\left(\text { for } s \gg \beta^{*}\right) \simeq \frac{s^{2}}{\beta^{*}} .
$$

Consequently by making a gradient change in the low- $\beta$ insertion quadrupoles and measuring the resulting tune shift, the $\beta$ value at the quadrupole can be evaluated. Knowing this value and the distance to the IP allows calculation of the $\beta$ value at the collision point [19]. In practice, in LEP the $\beta$ values at all eight superconducting quads are measured and the results are fed into an algorithm which allows 


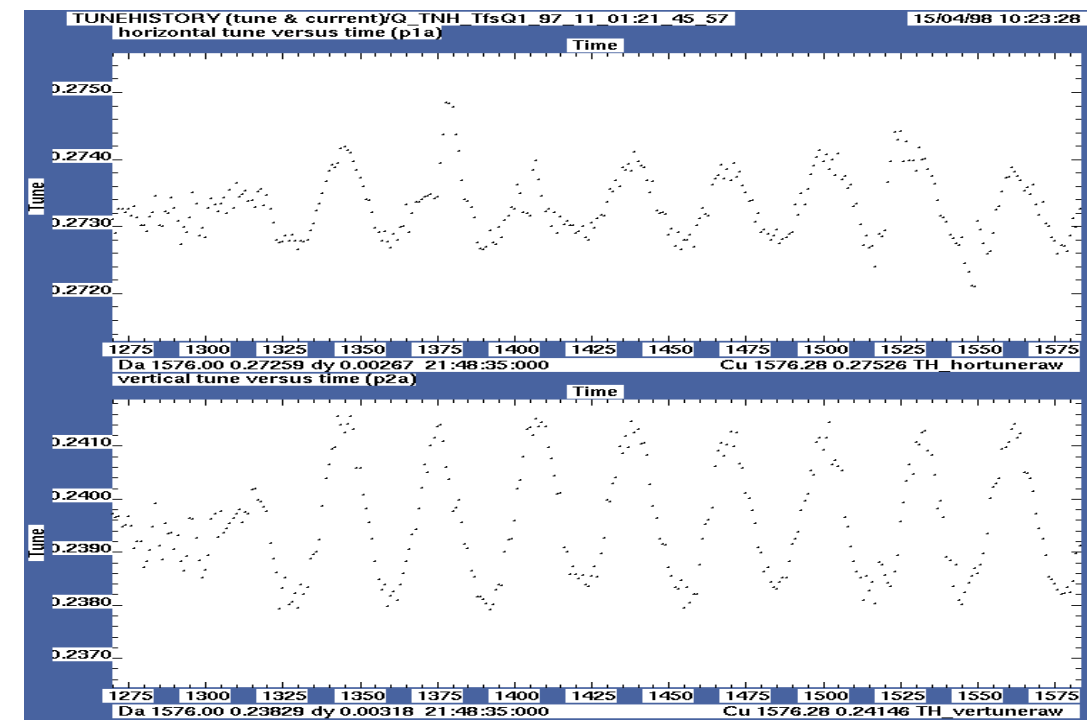

Figure 19: Measurement of chromaticity

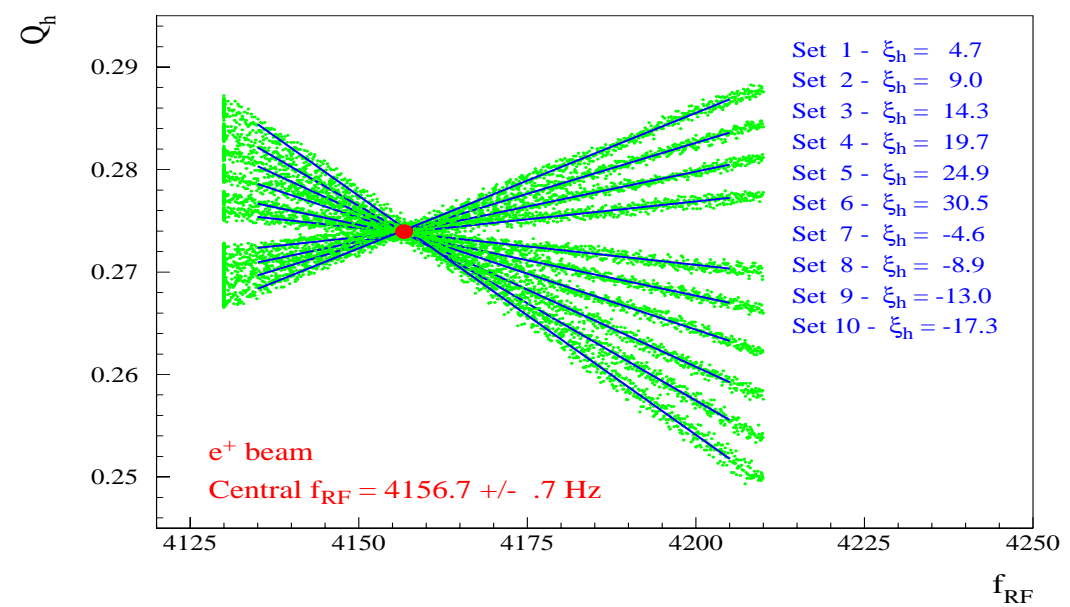

Figure 20: Measurement of the central frequency 


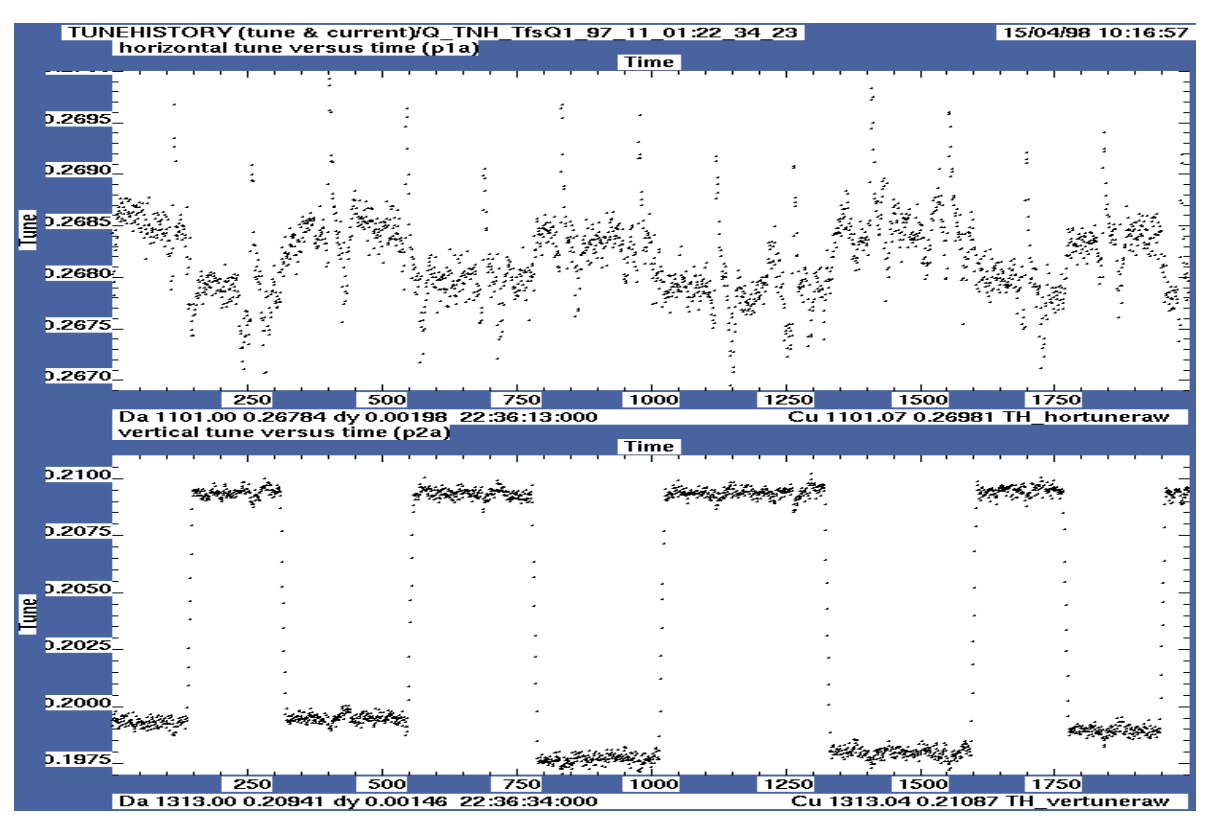

Figure 21: Measurement of the vertical beta at the interaction quads

correction of all four IPs to their nominal values $\left(\beta_{y}^{*}=5 \mathrm{~cm}\right)$. An example of such a measurement is shown in Figure 21. The unperturbed tune value in this plot is around 0.209 and each time a change in the gradient is made the tune value decreases by about 0.01 . The step functions in tune correspond to the introduction of the $\Delta k$ and returning to zero. The different levels of $\Delta Q$ correspond to the different interaction regions. Using this technique allows a precision and equality of around $\pm 2 \%$ in all four interaction regions. The equality of the tune shift caused by the quads left and right of the IP also allow evaluation of the symmetry of the $\beta$ functions and thus the azimuthal location of the minimum $\beta$.

\subsection{Q loop}

An obvious extension of the continuous measurement of the frequency response of the beam is to close the loop and control the beam frequency.

This is done in the transverse plane in LEP by the "Q loop", which simply compares the output frequency of the phase-lock loop with a reference frequency and uses the difference to control the excitation of quadrupoles [20]. In this way the tune of the beam is controllable. The reliable operation of the Q loop has become essential to the loss-free energy ramping of high intensity beams.

\section{Emittance measurement}

In LEP operation, the beam sizes are measured by two devices both using the synchrotron light emitted by the beam. The light in the near ultra-violet range has been used since the early commissioning days to give a real time image of the beam [21]. More recently and at higher energies light in the x-ray range has been used to give an accurate measure of the beam size. Clearly from equation (3), in order to measure the emittance the second term $\left(D \frac{\sigma_{E}}{E}\right)$ must be zero or known and the $\beta$ function must be known at the location of the synchrotron light monitor. In practice this implies that two locations are needed for the light monitors, one where the dispersion is vanishing and the other where the dispersion is high. Consequently, the measurement of $\beta$ and $D$ is essential for achieving the best precision for the emittance and energy spread evaluated from the measured beam sizes. Figure 22 shows a series of emittance measurements as a function of the RF frequency. Changing the RF frequency is used operationally to control the emittance i.e. 


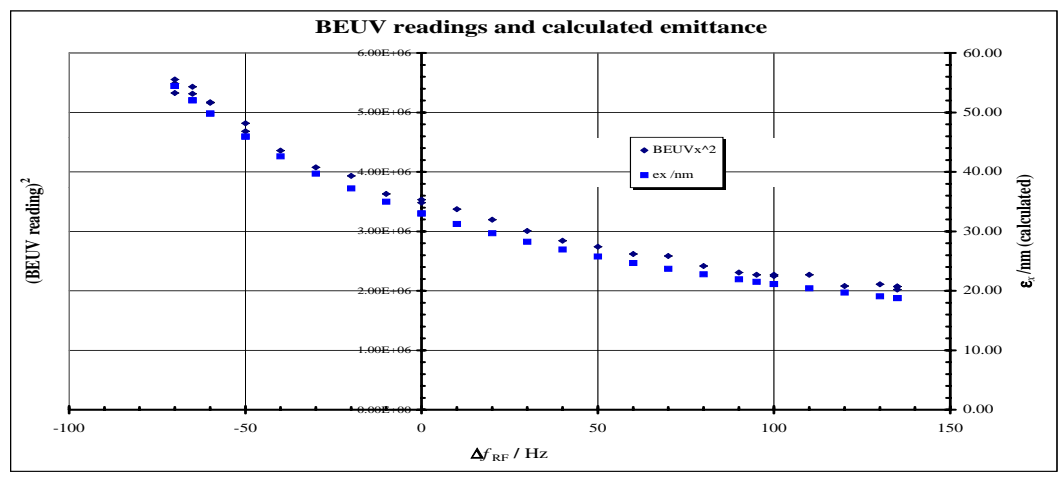

Figure 22: Measurement of the horizontal emittance during Jx variation using the synchrotron-light monitor

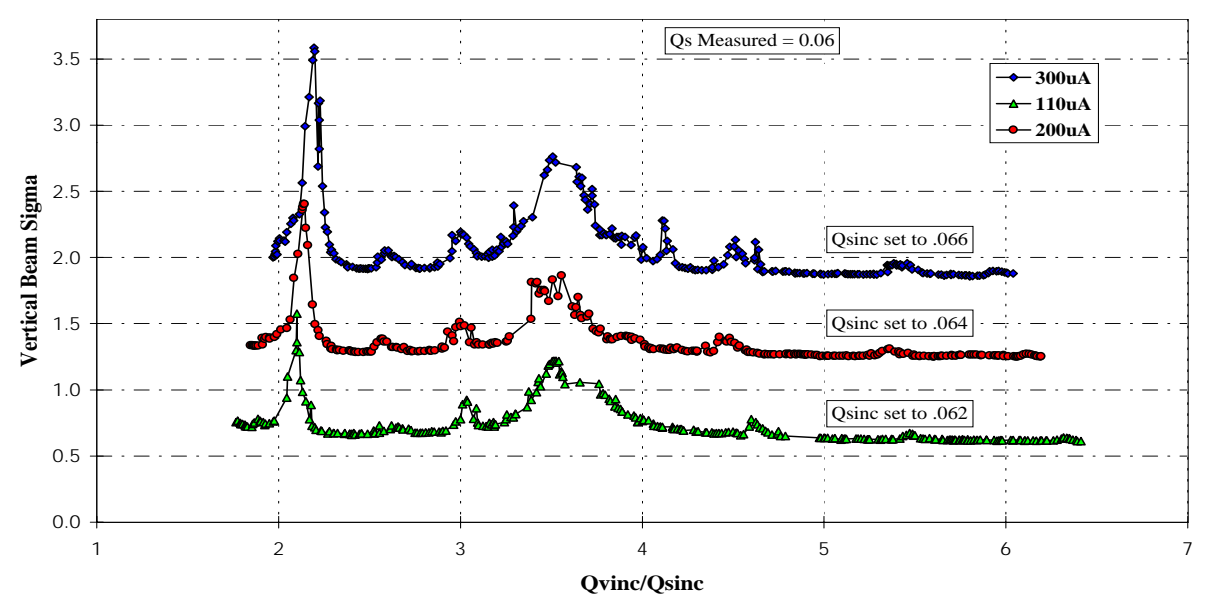

Figure 23: Measurement of synchro-betatron resonances.

$$
\epsilon_{x}=\frac{\sigma_{x}^{2}}{\beta_{x}} \propto \frac{1}{J_{x}} \simeq \frac{1}{J_{x 0}+\Delta J_{x}} \text { and } \Delta J_{x}=J^{\prime} \frac{\Delta p}{p}=-\frac{J^{\prime}}{\eta} \frac{\Delta f_{r f}}{f_{r f}}
$$

The synchrotron light monitors have been used extensively both qualitatively and quantitatively in LEP since the first circulating beam was established. These devices are invaluable for the continuous optimization of the machine performance.

\subsection{Tune scans}

Continuous measurement of the transverse beam sizes is extremely useful during physics as a tool for maximizing the luminosity by reducing the beam size. Another important application is the measurement of beam size as a function of a variable parameter such as the machine tune. For this reason an automatic tool was developed [22] for variation of the betatron tunes and simultaneous measurement of various parameters such as the beam size and the lifetime. This tool was used extensively for measuring the strength of resonances and for searching for the optimum regions in tune space.

Figure 23 shows a measurement [23] of the vertical beam size as a function of the vertical tune normalized to the synchrotron tune. From these results one clearly identifies several synchro-betatron resonances as well as the main coupling resonance. This technique was used to measure and minimize the strengths of many resonances by variation of the appropriate parameters. 


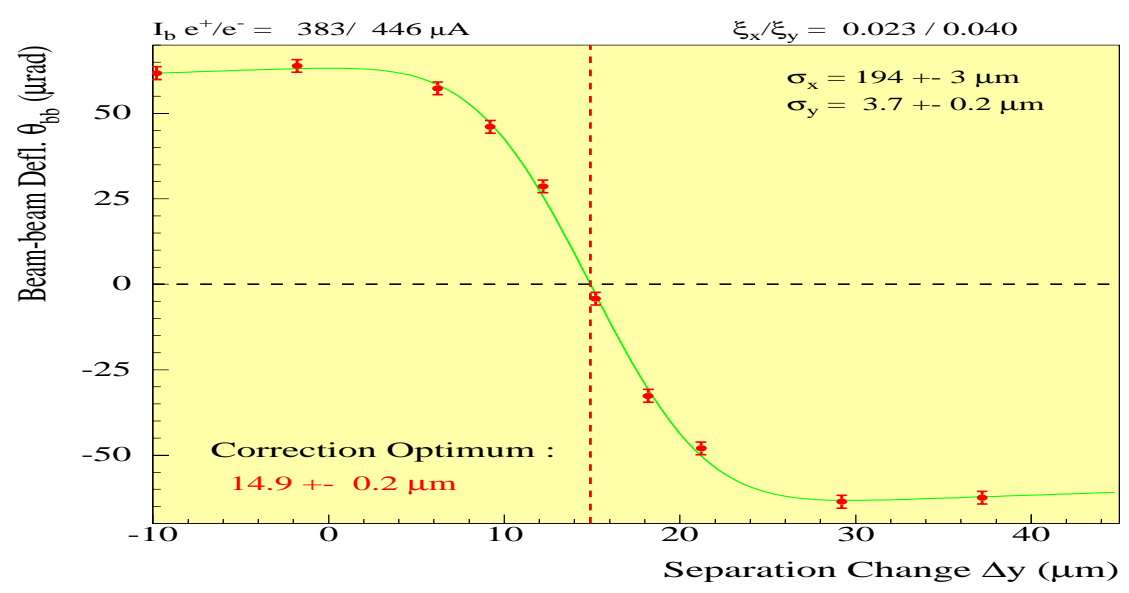

Figure 24: Measurement of angular deflections due to beam-beam forces

\section{Measurements in collision}

\subsection{Beam-beam deflection}

It is clear from equation (2) that if the electron and positron beams are not colliding perfectly "head-on" then a loss of luminosity results. In the early days of operation the beams were steered into collision by the electro-static separators using the signals from the luminosity monitors. However due to the slow time response of these monitors the procedure was time consuming and therefore wasteful of luminosity. The technique which is used now measures the angular deflection produced on one beam by the electromagnetic force associated with the other beam [24], [25], [26]. The electro-magnetic deflection exerted by the counter rotating beams on each other is a highly non-linear odd function that traverses zero when the separations between the beams is zero. If it is assumed that the charge distribution is and remains an unchanged Gaussian throughout the measurement, then there is an analytical expression relating the angular deflection to the separations. The angular deflection is measured using two pick-ups on either side of the interaction point, and the separations are generated by the electro-static separators. The measured values are then fitted to the analytical expression with four free parameters. Figure 24 shows an example of measured data with the fit. This technique is very rapid and reproducible and is therefore used operationally on every fill of LEP.

\section{Acknowledgments}

Sincere thanks are due to C. Bovet, H. Burkhardt, P. Collier, B. Dehning, J. Jowett, R. Jung, M. Lamont, G. Morpurgo, A. Müller, M. Placidi, J. Poole, and J. Wenninger for their help in preparing the presentation as well as this paper.

\subsubsection{References}

\section{References}

[1] S. Myers; "LEP2 : Present and Future Performance and Limitations", Proc. of the 1997 Particle Accelerator Conference: PAC '97 Vancouver, Canada ; 12 - 16 May 1997, also CERN-SL-97-022DI .

[2] S. Myers; "LEP Status and Plans", Proc. of the US Particle Accelerator Conference and the 16th Int. Conf. on High Energy Accelerators, Dallas USA, (1995). 
[3] A. Hofmann; "Performance Limitations in LEP", Proc. of the Fourth European Particle Accelerator Conference, London, 27 June - 1 July, 1994. (pp 73-77), (1994).

[4] S. Myers; "LEP Performance and Plans", Proc. 15th Int. Conf. on High Energy Accelerators, Hamburg 1992; HEACC92, p. 66, (1992).

[5] K. Unser: "Design and Preliminary Tests of a Beam Intensity Monitor for LEP", Proc. of the 1989 Particle Accelerator Conference : PAC '89 Chicago, USA, March 1989, also CERN-LEP BI/89-07.

[6] A. Burns et al.; "Real Time Monitoring of LEP Beam Currents and Lifetime", Proc. of the Fourth European Particle Accelerator Conference, London, 27 June - 1 July, 1994. (pp 73- 77), (1994) and CERN SL/94-28(BI), June 1994.

[7] G. Morpurgo; "The Software for the CERN LEP Orbit Measurement System", CERN SL/91-41, 1991.

[8] I. Barnett et al.;"Dynamic Beam Based Alignment”, CERN SL/94-84, July 1995.

[9] B. Dehning et al.;"Dynamic Beam Based Calibration of Beam Position Monitors", Proc. of the Sixth European Particle Accelerator Conference : EPAC '98 Stockholm, Sweden ; 22 - 26 June 1998, also CERN SL-98-038 (BI).

[10] D. Brandt et al.; "A New Closed Orbit Correction Procedure for the CERN SPS and LEP”, Nuclear Instruments and Methods, A293 (1990), p305.

[11] J. Borer et al., "Harmonic analysis of coherent bunch oscillations in LEP", Proc. of the 3rd EPAC, Berlin, 24-28 March 1992, pp. 1082-4., and CERN SL/92-15 (DI).

[12] A. Burns et al.; "Betatron Function Measurement at LEP using the BOM 1000 Turns Facility", 1993 Particle Accelerator ConferenceWashington, DC, USA; 17-20 May 1993 (2103-2105), also CERN SL 93-20 (DI).

[13] P. Castro-Garcia, "Luminosity and beta function measurement at the electron-positron collider ring LEP”, PhD Thesis, CERN SL/96-70 (BI).

[14] J.M. Jowett; "Non-Linear Resonances; Predictions, Effects and Measurements", Proceedings of the Seventh LEP Performance Workshop held at Chamonix, France, 1997, CERN SL/97-06.

[15] G. Morpurgo, "The BOM 1000-turn display: a tool to visualize the transverse phase-space topology at LEP”, Proc. of the 6th EPAC, Stockholm, 22-26 June 1998, pp. 1571-3, and CERN SL-98045 (BI).

[16] A. Müller and J. Wenninger; "Measurements of Coherent Damping and Tune Shifts with Amplitude at LEP" , Proc. of the 6th European Particle Accelerator Conference : EPAC '98 Stockholm, Sweden ; 22-26 June 1998.

[17] G. Buur et al., "Dynamic Tune and Chromaticity Measurements in LEP”, Proc. 3rd EPAC, Berlin, 24-26 March 1992, pp. 1043-5, and CERN SL/92-15 (DI).

[18] H. Schmickler; "Measurement of the Central Frequency in LEP", CERN SL-MD Note 89.

[19] C. Bovet and C. Fischer, "Precise Determination of the Amplitude Function in Collider Insertions with an Application to LEP”, Proc. Of the IEEE PAC, Chicago, March 20-23, 1989, pp. 1301-3, and CERN/LEP-BI/89-05.

[20] O. Berrig et al., "The Q-loop: A Function Driven Feedback System for the Betatron Tunes during the LEP Energy Ramp", Proc. 6th EPAC, Stockholm, 22-26 June 1998, pp. 1717-9, and CERN SL-98-039 (BI).

[21] R. Jung, "Precision Emittance Measurements in LEP with Imaging Telescopes, Comparison with Wire Scanner and X-Ray Detector Measurements", KEK Proc. 95-7, A, Sept. 1995, and CERN SL/95-63 (BI). 
[22] H. Schmickler, “Tune scans in LEP”, CERN SL/95-67 (BI).

[23] S. Myers; "Measurement of the Parameter Dependence of Synchro-Betatron Resonances in LEP", Proc. of the International Workshop on Particle Dynamics in Accelerators, Tsukuba, Japan 21-26 Nov. 1994, KEK Proc. 95-7 (97-104).

[24] J. Wenninger; "Measurement of the Beam-Beam Deflections at the Interaction Points of LEP", CERN-SL/Note 96-01.

[25] C. Bovet et al.; "Luminosity Optimization using Beam-beam Deflections at LEP", Proc. of the Fifth European Particle Accelerator Conference, Sitges, Spain, June 1996.

[26] M. Placidi and J. Wenninger, "Interaction Region Diagnostics in e+e- Ring Colliders", Proc. ICFA Workshop on Beam Dynamics Issues for e+e- Factories, Frascati, 20-25 October 1997, and CERNSL/98-13 (BI). 